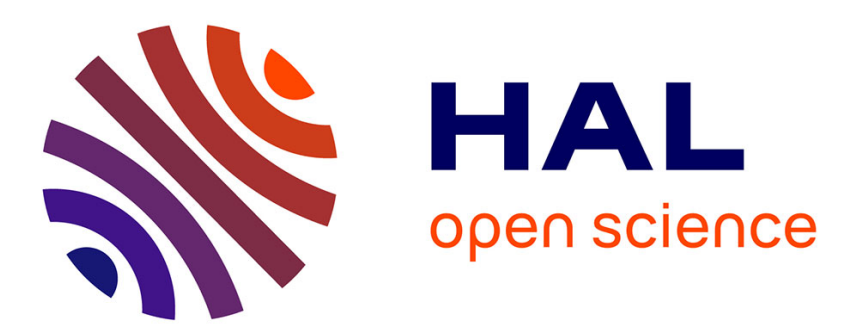

\title{
A new dynamic substructuring method for nonlinear and dissipative systems
}

Colas Joannin, Benjamin Chouvion, Fabrice Thouverez

\section{To cite this version:}

Colas Joannin, Benjamin Chouvion, Fabrice Thouverez. A new dynamic substructuring method for nonlinear and dissipative systems. ECCOMAS Congress 2016 - VII European Congress on Computational Methods in Applied Sciences and Engineering, Jun 2016, Hersonissos, Greece. hal-01389701

\section{HAL Id: hal-01389701 \\ https://hal.science/hal-01389701}

Submitted on 29 Oct 2016

HAL is a multi-disciplinary open access archive for the deposit and dissemination of scientific research documents, whether they are published or not. The documents may come from teaching and research institutions in France or abroad, or from public or private research centers.
L'archive ouverte pluridisciplinaire HAL, est destinée au dépôt et à la diffusion de documents scientifiques de niveau recherche, publiés ou non, émanant des établissements d'enseignement et de recherche français ou étrangers, des laboratoires publics ou privés. 


\title{
A NEW DYNAMIC SUBSTRUCTURING METHOD FOR NONLINEAR AND DISSIPATIVE SYSTEMS
}

\author{
Colas Joannin ${ }^{1,2}$, Benjamin Chouvion ${ }^{2}$, and Fabrice Thouverez ${ }^{2}$ \\ ${ }^{1}$ Turbomeca, Safran group \\ Avenue Joseph Szydlowski, 64510 Bordes, France \\ e-mail: colas.joannin@doctorant.ec-lyon.fr \\ ${ }^{2}$ Laboratoire de Tribologie et Dynamique des Systèmes, École Centrale de Lyon \\ 36 avenue Guy de Collongue, 69134 Écully, France \\ e-mail: \{benjamin.chouvion,fabrice.thouverez\}@ec-lyon.fr
}

Keywords: Reduced-Order Model, Nonlinear Mode, Component Mode Synthesis, Mistuning.

\begin{abstract}
This work is devoted to the development of a new dynamic substructuring method inspired by classic fixed-interface component mode synthesis, in order to compute the steadystate vibrations of dissipative, nonlinear structures. For each substructure, the displacement field is sought as a multiharmonic oscillation made of standard static mode shapes, supplemented by the eigenvectors of the nonlinear complex modes of the substructure computed with fixed-boundary conditions. The method eventually leads to a strongly reduced nonlinear algebraic system, easily solved by iterative solvers. The procedure is tested on a lumped parameter model of bladed disk subjected to dry friction nonlinearities, with or without structural mistuning, and proves very efficient in terms of computational cost. These results emphasize the promising capabilities of this new reduced-order modeling technique to tackle such nonlinear systems exhibiting high modal density.
\end{abstract}




\section{INTRODUCTION}

Reduced-order modeling has always been a key problematic in computational mechanics. Despite the soaring hardware and software capabilities offered by modern electronics and computing, finite element analysis of large scale industrial systems remains challenging when some complex and nonlinear phenomena are to be included in the design process. Component mode synthesis (CMS) methods [1, 2, 3, 4] have been widely used in structural dynamics, but can unfortunately prove ill-suited to the study of nonlinear systems when a large number of degreesof-freedom (DOF) are impacted by strong nonlinear effects. For such systems, standard techniques may indeed fail to find a satisfying compromise between order-reduction and accuracy, due to the lack of representativity of the reduction basis. From this perspective, owing to their ability to capture the essence of the nonlinearities [5, 6], nonlinear complex modes are interesting candidates in devising new effective and efficient approaches to reduce nonlinear models.

In bladed-disk dynamics, the loss of cyclic symmetry of the structures, referred to as mistuning, has been a leading subject of research, first tackled by a few authors in the 1960s [7, 8, 9, 10] and widely studied since then [11]. This phenomenon mainly denotes the discrepancies between the blades of the assembly, resulting for instance from the manufacturing process and in-operation wear, but which can also be intentionally introduced in the nominal design. Even though the mistuning of cyclic structures is known to potentially induce a significant increase in the vibration level, it has also proved, indeed, to improve the robustness of the design against hazardous instabilities such as flutter. In order to perform quantitative analyses on industrial models, several mistuning dedicated reduced-order modeling techniques have been devised to efficiently handle large scale systems [12, 13, 14, 15]. Unfortunately, most of them are not applicable in the presence of nonlinearities, hence the need for further endeavours devoted to the reduced-order modeling of nonlinear, mistuned cyclic structures.

In the present paper, a new approach is proposed to build reduced-order models of nonlinear and dissipative systems. The method does not require any cyclic or nearly-cyclic symmetry assumption, and can thus be directly applied to the study of strongly mistuned bladed-disks. The procedure mimics a standard Craig-Bampton CMS, but makes use of the notion of nonlinear complex modes to build nonlinear superelements, including all internal nonlinear DOFs in the reduction basis. The resulting algebraic system can thus be much smaller than that of a standard harmonic balance method (HBM), which requires to retain all nonlinear DOFs as master coordinates. The flexibility of nonlinear complex modes [5, 6, 16] makes this reduction technique suitable for the study of a broad range of nonlinearities, dissipative and non-smooth ones included.

In Section 2, the main steps of the computation of nonlinear complex modes are first reminded, and the nonlinear superelement equations are derived. A lumped-parameter model of bladed-disk exhibiting dry friction nonlinear elements is then used in Section 3 to illustrate the capability of this new technique. First, the method is tested on a tuned system, and is then used to synthesise the response after application of a random mistuning pattern. Section 4 presents the performance of the procedure, and its potential limitations. 


\section{NONLINEAR COMPONENT MODE SYNTHESIS}

This section aims at summarizing the main steps to build a nonlinear superelement. The computation of nonlinear complex modes is briefly reminded in Section 2.1, and the notion is illustrated on a basic 2-DOFs system. In Section 2.2, the system of equations defining a nonlinear superelement are then derived. As mentioned in the introduction, this new reducedorder modeling procedure shares some similarities with classic CMS methods, and is hereafter referred to as CNCMS, for component nonlinear complex mode synthesis.

\subsection{Nonlinear complex modes}

The concept of nonlinear mode is nowadays well established in the community of nonlinear dynamicists. A comprehensive review listing all major contributions and techniques applicable to structural dynamics is proposed in [17]. Nonlinear complex modes are an extension of nonlinear normal modes to dissipative systems, that aims at taking advantage of numerical and frequency-based formulation such as the harmonic balance method to compute the free vibrations of damped nonlinear structures. First introduced in [5], they have proved tremendously interesting to study all sorts of nonlinear systems [6, 16, 18], and are fundamentals to the reduced-order modeling technique presented here. Only a brief reminder is provided here to introduce some notations, but more details can be found in [5, 6, 16, 18].

Nonlinear complex modes refer to pure or pseudo-oscillations of the system of nonlinear differential equations (1). The terms are defined in a classic manner, with $\mathbf{M}$ the mass matrix, $\mathbf{C}$ the viscous damping matrix, $\mathbf{K}$ the linear stiffness matrix, and $\mathbf{f}_{n l}$ a term of nonlinear restoring and dissipative forces.

$$
\mathbf{M} \ddot{\mathbf{x}}(t)+\mathbf{C} \dot{\mathbf{x}}(t)+\mathbf{K} \mathbf{x}(t)+\mathbf{f}_{n l}(\mathbf{x}, \dot{\mathbf{x}})=\mathbf{0}
$$

The $\mathrm{n}$-th mode $\mathbf{x}_{n}$ is approximated by a truncated series of damped oscillations of fundamental frequency $\omega_{n}$ and modal damping $\beta_{n}$, such that with $\lambda_{n}=-\beta_{n}+i \omega_{n}$ and c.c. referring to the complex conjugate terms,

$$
\mathbf{x}_{n}(t)=\frac{1}{2}\left\{\sum_{k=1}^{n_{h}} \hat{\mathbf{x}}_{n, k} e^{k t \lambda_{n}}+\text { c.c. }\right\}
$$

Similarly to standard Galerkin methods, the approximation (2) is then substituted into the governing equations (1), and the orthogonality of the residual to the subspace spanned by the functions $\left\{e_{k}=e^{i k t \omega_{n}}\right\}, \forall k \in \llbracket 1, n_{h} \rrbracket$, is enforced, with respect to the inner product $(3)$ where $\overline{g(t)}$ is the complex conjugate of $g(t)$. In order to take advantage of the orthogonality property of the complex exponential functions, the solution is assumed to be undamped over the period $T$ used in the inner product, which has proved to have a negligible effects on the accuracy of the results (see [5] for further information).

$$
\langle f \mid g\rangle=\frac{2}{T} \int_{0}^{T} f(t) \overline{g(t)} d t, \text { with } T=\frac{2 \pi}{\omega_{n}}
$$

The resulting algebraic system (4) is the eigenproblem corresponding to the nonlinear complex mode $\mathbf{x}_{n}$, where the term $\left\langle\mathbf{f}_{n l} \mid e_{k}\right\rangle$ potentially couples all the equations. This system is underdetermined and must be supplemented with two equations, classically in the form of a 
phase condition and a continuation scheme, which are not detailed here. The interested reader is referred to [18] for further information.

$$
\begin{aligned}
& \forall k \in \llbracket 1, n_{h} \rrbracket: \\
& {\left[\left(k \lambda_{n}\right)^{2} \mathbf{M}+\left(k \lambda_{n}\right) \mathbf{C}+\mathbf{K}\right] \hat{\mathbf{x}}_{n, k}+\left\langle\mathbf{f}_{n l} \mid e_{k}\right\rangle=\mathbf{0}}
\end{aligned}
$$

The flexibility of nonlinear complex modes arises, among other things, from their applicability to a broad range of nonlinearities, including non-smooth forces. This is made possible and easy to implement by means of the alternating frequency-time scheme (AFT) proposed in [19], and widely used in conjunction with harmonic balance methods [5, 16, 18]. This procedure uses direct $(\mathcal{F})$ and inverse $\left(\mathcal{F}^{-1}\right)$ Fourier transforms to compute $\left\langle\mathbf{f}_{n l} \mid e_{k}\right\rangle$ from the time-domain definition of the nonlinear terms.

To exemplify nonlinear complex modes, let us now consider the academic system on Figure 1 subjected to a dry friction force, with the lumped-parameters taking their values in Table 1 . Figure 2 shows the variations of the first natural frequency and corresponding modal damping as a function of the displacement amplitude $\left|x_{1}\right|$. These so-called backbones are thoroughly explained in previous papers [5, 6, 18] and will not be discussed here. However, it should be remembered in the following sections that nonlinear complex modes are functions of a parameter describing the intensity of the nonlinear effects, here the amplitude of vibration $\left|x_{1}\right|$, and that the modes of friction-damped systems exhibit an optimum damping point, corresponding to a maximum dissipation arising from the nonlinearity.

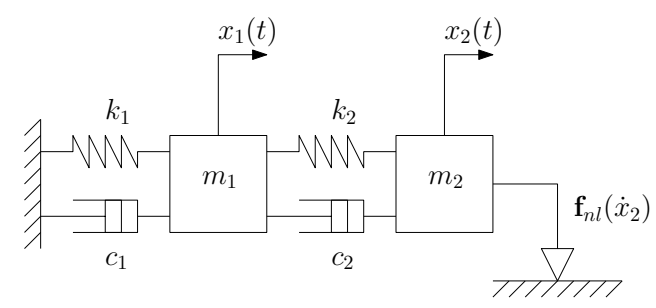

Figure 1: 2-DOFs dry-friction damped model

\begin{tabular}{lccc}
\hline- (unit) & $\mathrm{m}(\mathrm{kg})$ & $\mathrm{c}(\mathrm{Ns} / \mathrm{m})$ & $\mathrm{k}(\mathrm{N} / \mathrm{m})$ \\
\hline DOF1 & 1 & 0.5 & 640 \\
DOF2 & 0.02 & 0.5 & 40 \\
\hline
\end{tabular}

Table 1: Values of the lumped-parameters for the 2-DOFs system

\subsection{Nonlinear superelement}

This section is dedicated to the derivation of the equations governing one superelement, built by means of nonlinear complex modes. Each substructure being treated indepedently, the nonlinear superelement could be perfectly used in conjunction with standard CMS superelements 

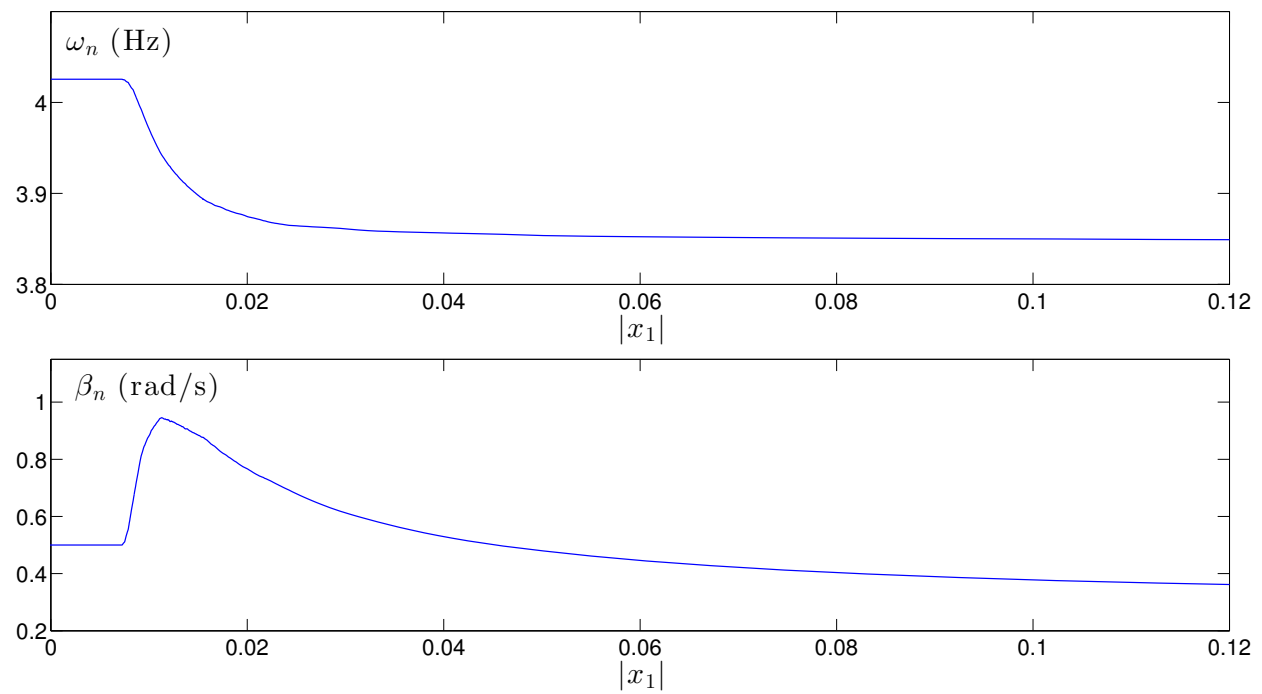

Figure 2: Nonlinear natural frequency and modal damping of the 2-DOFs model

using linear modes. This reduced-order model is devoted to the study of forced vibrations arising from harmonic or multi-harmonic excitations, of the form given in Eq. (5) where $\Omega$ is the fundamental forcing frequency.

$$
\mathbf{f}_{e}(t)=\frac{1}{2}\left(\sum_{k}\left\langle\mathbf{f}_{e} \mid e_{k}\right\rangle e^{i k \Omega t}+\text { c.c. }\right)
$$

The displacement of the substructure is sought as a multi-harmonic oscillation (6) made of nonlinear eigenvectors $\boldsymbol{\varphi}_{n, k}$ corresponding to fixed-interface nonlinear complex modes, supplemented by linear static modes $\boldsymbol{\psi}_{s, k}$ as in a standard fixed-interface CMS [1]. The generalized coordinates $q_{n}$ and $p_{s}$ corresponding to the nonlinear complex modes and static modes, respectively, are the new unknowns of the problem.

$$
\mathbf{x}(t)=\frac{1}{2}\left(\sum_{n} q_{n} \sum_{k} \boldsymbol{\varphi}_{n, k}\left(\left|q_{n}\right|\right) e^{i k \Omega t}+\sum_{s} p_{s} \sum_{k} \boldsymbol{\psi}_{s, k} e^{i k \Omega t}+\text { c.c. }\right)
$$

The relation between the nonlinear eigenvectors $\varphi_{n, k}$ and the k-th harmonics $\hat{\mathbf{x}}_{n, k}$ in Eq. (2) is given by Eq. (7).

$$
\hat{\mathbf{x}}_{n, k}=q_{n} \boldsymbol{\varphi}_{n, k}
$$

In the following, the vectors $\boldsymbol{\psi}_{s, k}$ are all taken equal to the standard static modeshapes of a Craig-Bampton CMS, given by Eq. (8) when the DOFs are partitionned into a set of internal (subscript $i$ ) and boundary (subscript $b$ ) DOFs, although different vectors could be used.

$$
\begin{aligned}
& \forall k \in \llbracket 1, n_{h} \rrbracket: \\
& \boldsymbol{\psi}_{s, k}=-\mathbf{K}_{i i}^{-1} \mathbf{K}_{i b} \quad \text { with } \quad \mathbf{K}=\left[\begin{array}{cc}
\mathbf{K}_{i i} & \mathbf{K}_{i b} \\
\mathbf{K}_{b i} & \mathbf{K}_{b b}
\end{array}\right]
\end{aligned}
$$


As mentioned in Section 2.1 for the 2-DOFs model, the modal parameters $\varphi_{n, k}$ and $\lambda_{n}$ are functions of a parameter describing the activation of the nonlinearity. In Eq. (6), this parameter is the amplitude $\left|q_{n}\right|$ of the coordinate of the corresponding mode. In theory, since the mode is complex, two variables should be used to describe its variations (i.e. the real and imaginary parts of $q_{n}$ ), but it was shown in [5] that chosing only the amplitude $\left|q_{n}\right|$ yields excellent results for dry-friction nonlinearities. In the following equations, the dependency of $\varphi_{n, k}$ and $\lambda_{n}$ on $\left|q_{n}\right|$ is implied.

In accordance with Galerkin methods, the approximation (6) is substituted into Eq. (1), after addition of the external forcing $\mathbf{f}_{e}$ from Eq. (5) on the right hand side, and the residual is orthogonalized to the subspace spanned by the functions $\left\{e_{k}=\mathrm{e}^{i k \Omega t}\right\}$ with respect to the inner product (3). The operation yields,

$$
\begin{aligned}
& \forall k \in \llbracket 1, n_{h} \rrbracket: \\
& {\left[(i k \Omega)^{2} \mathbf{M}+(i k \Omega) \mathbf{C}+\mathbf{K}\right]\left\{\sum_{n} q_{n} \boldsymbol{\varphi}_{n, k}+\sum_{s} p_{s} \boldsymbol{\psi}_{s, k}\right\}+\left\langle\mathbf{f}_{n l} \mid e_{k}\right\rangle=\left\langle\mathbf{f}_{e} \mid e_{k}\right\rangle}
\end{aligned}
$$

As performed in [6] in a modal synthesis procedure, the term $\left\langle\mathbf{f}_{n l} \mid e_{k}\right\rangle$ is replaced by the sum of the analogous vectors in the eigenproblem (4), which yields the set of equations (10), where the second line accounts now for $\left\langle\mathbf{f}_{n l} \mid e_{k}\right\rangle$. The matrix $\tilde{\mathbf{K}}$ differs from the matrix $\mathbf{K}$ owing to the fixed boundary conditions enforced during the computation of the modes.

$$
\begin{aligned}
& \forall k \in \llbracket 1, n_{h} \rrbracket: \\
& \sum_{n}\left[(i k \Omega)^{2} \mathbf{M}+(i k \Omega) \mathbf{C}+\mathbf{K}\right] q_{n} \boldsymbol{\varphi}_{n, k} \\
- & \sum_{n}\left[\left(k \lambda_{n}\right)^{2} \mathbf{M}+\left(k \lambda_{n}\right) \mathbf{C}+\tilde{\mathbf{K}}\right] q_{n} \boldsymbol{\varphi}_{n, k} \\
+ & \sum_{s}\left[(i k \Omega)^{2} \mathbf{M}+(i k \Omega) \mathbf{C}+\mathbf{K}\right] p_{s} \boldsymbol{\psi}_{s, k} \\
- & \left\langle\mathbf{f}_{e} \mid e_{k}\right\rangle \approx \mathbf{0}
\end{aligned}
$$

Finally, the residuals $(10)$ are orthogonalized to the subspace spanned by the basis vectors $\varphi_{n, k}$ and $\boldsymbol{\psi}_{s, k}$ with respect to the classic Hermitian form in $\mathbb{C}^{n}$. The whole process leads to the set of algebraic equations 11 governing the motion of a substructure, where $\bullet^{\dagger}$ refers to the Hermitian transpose.

$$
\begin{aligned}
& \forall k \in \llbracket 1, n_{h} \rrbracket: \\
& \boldsymbol{\rho}_{k}=\left[\begin{array}{cc}
\boldsymbol{\Phi}_{n, k}^{\dagger}\left(\mathbf{Z}_{k} \boldsymbol{\Phi}_{n, k}-\boldsymbol{\xi}_{k}\right) & \boldsymbol{\Phi}_{n, k}^{\dagger} \mathbf{Z}_{k} \boldsymbol{\Psi}_{s, k} \\
\boldsymbol{\Psi}_{s, k}^{\dagger}\left(\mathbf{Z}_{k} \boldsymbol{\Phi}_{n, k}-\boldsymbol{\xi}_{k}\right) & \boldsymbol{\Psi}_{s, k}^{\dagger} \mathbf{Z}_{k} \boldsymbol{\Psi}_{s, k}
\end{array}\right]\left\{\begin{array}{l}
\mathbf{q} \\
\mathbf{p}
\end{array}\right\}-\left\{\begin{array}{c}
\boldsymbol{\Phi}_{n, k}^{\dagger}\left\langle\mathbf{f}_{e} \mid e_{k}\right\rangle \\
\boldsymbol{\Psi}_{s, k}^{\dagger}\left\langle\mathbf{f}_{e} \mid e_{k}\right\rangle
\end{array}\right\}=\mathbf{0}
\end{aligned}
$$

with $\mathbf{Z}_{k}=\left[(i k \Omega)^{2} \mathbf{M}+(i k \Omega) \mathbf{C}+\mathbf{K}\right]$ and $\boldsymbol{\xi}_{k}=\left[\mathbf{M} \boldsymbol{\Phi}_{n, k} \boldsymbol{\Lambda}_{n}^{2} k^{2}+\mathbf{C} \boldsymbol{\Phi}_{n, k} \boldsymbol{\Lambda}_{n} k+\tilde{\mathbf{K}} \boldsymbol{\Phi}_{n, k}\right]$ the dynamic stiffness matrix and the term arising from the substitution of each $\left\langle\mathbf{f}_{n l} \mid e_{k}\right\rangle$ from the eigenproblems, $\boldsymbol{\Phi}_{n, k}$ and $\boldsymbol{\Psi}_{s, k}$ the matrices made of the column vectors $\boldsymbol{\varphi}_{n, k}$ and $\boldsymbol{\psi}_{s, k}, \boldsymbol{\Lambda}_{n}$ a diagonal matrix made of the nonlinear eigenvalues distributed along the diagonal, and $\mathbf{q}$ and $\mathbf{p}$ the column vectors listing the general coordinates $q_{n}$ and $p_{s}$ respectively. 
The reduced-order order model of the global system is eventually built by coupling the substructures through the generalized coordinates corresponding to the static modeshapes, $p_{s}$. If more than one harmonic is kept in the series expansion (2), which is typically the case for strong and non-smooth nonlinearities such as contact and friction, the resulting system is overdetermined. However, one could, as a first approximation, discard the equations for which $\left\langle\mathbf{f}_{e} \mid e_{k}\right\rangle=\mathbf{0}$, which has proved to have a neglictible impact on the accuracy of the results. In the case of a mono-harmonic excitation, as in Section 3, the resulting system of equations is thus square.

Whether the system dealt with is over-determined or square, it is clearly nonlinear due to the dependency of the modes over the amplitude of their coordinates $\left|q_{n}\right|$, and must be solved iteratively by standard routines available in most commercial computing softwares. In practice, $\boldsymbol{\varphi}_{n, k}$ and $\lambda_{n}$ are known for discrete values of $\left|q_{n}\right|$ from the nonlinear mode computation performed beforehand, and it requires now that they be interpolated at each iteration of the solver, which is readily achieved by linear or cubic interpolations.

\section{NUMERICAL EXAMPLES}

The dynamic substructuring method derived in Section 2 is now tested on a lumped-parameter model of bladed-disk, first on a tuned system, and then on the mistuned model, in order to demonstrate its efficiency in dealing with such complex and rich systems.

\subsection{Lumped-parameter model}

The cyclic model devised in this section aims at being representative of the phenomenology encountered in bladed-disk dynamics. It consists of identical sectors such as shown on Figure 3, with structural parameters taking the values reported in Table 2. The viscous damping parameters are defined so as to set the linear modal damping ratio of the first mode to $0.1 \%$. Throughout the study, the maximum harmonic number $n_{h}$ is set to 5, which has proved to provide a sufficient accuracy for the purpose of such analyses, and the nonlinear force arising from the dry-friction element is modeled by a hyperbolic tangent law [18].

\begin{tabular}{|l|ccccc|}
\hline$-($ unit $)$ & Tip & Middle & Root & Disk & Ground \\
\hline $\mathrm{m}(\mathrm{kg})$ & 0.2 & 0.3 & 0.4 & 1.2 & - \\
$\mathrm{c}(\mathrm{Ns} / \mathrm{m})$ & 1.3 & 0.7 & 26.7 & 33.3 & 0.4 \\
$\mathrm{k}\left(10^{6} \cdot \mathrm{N} / \mathrm{m}\right)$ & 2 & 1 & 40 & 50 & 0.6 \\
\hline
\end{tabular}

Table 2: Values of the parameters for the fundamental sector

In order to obtain clear and legible response curves for the mistuned system in Section 3.3 , a model with only 12 sectors is used throughout Section 3.2 and Section 3.3 . However, the performance of the method are appraised in Section 4.1 for 72 sectors, so as to deal with a more significant number of nonlinear DOFs, and thus assess the practical capabilities of the CNCMS.

\subsection{Tuned system}

The superelement of one sector is built from one nonlinear complex mode and two static modeshapes. Of course, for this tuned case, cyclic boundary conditions could have been applied 


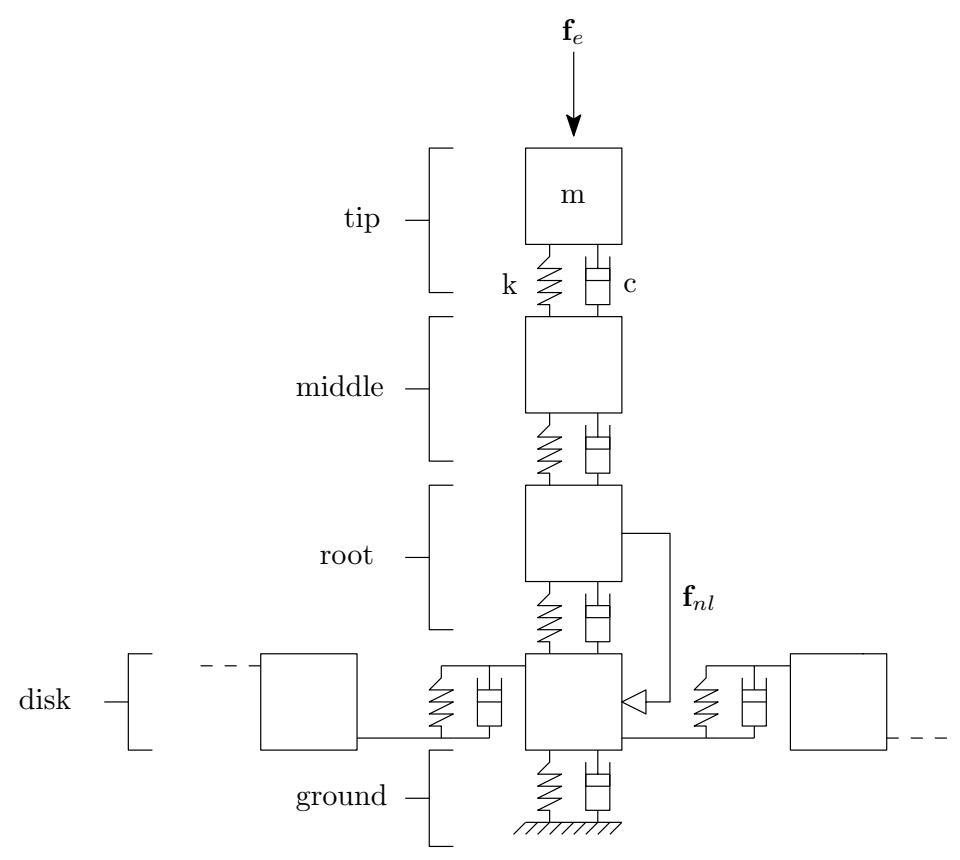

Figure 3: Fundamental sector of the cyclic model

to compute the mode, thus restricting the study to cyclic solutions. The system is first subjected at the blade tips to a travelling wave excitation, standard in bladed-disk dynamics, with 6 nodal parameters and for various excitation levels. In accordance with the linear theory of cyclic systems, the frequency response exhibits one resonance corresponding to the mode with 6 nodal diameters. Figure 4 shows the perfect agreement of the solution obtained by CNCMS with the reference, computed by means of a classic harmonic balance method (HBM), even for a strong activation of the nonlinearities. The shift of the resonance toward lower frequencies as the amplitude of the response increases is characteristic of friction damped systems, and is a direct consequence of the evolution of the nonlinear natural frequencies that can be observed on Figure 2 .

\subsection{Mistuned system}

An interesting application of the reduced-order modeling technique presented in this paper is the study of nonlinear and mistuned cyclic structures. In order to prove that the method can effectively handle such systems, the lumped-parameter model of Section 3.1 is modified by defining a new type of sector, with different tip and middle stiffness values, so as to shift the first natural frequency by $5 \%$ from that of the initial sector. The values taken by the structural parameters of this new sector are reported in Table 3. The fundamental sector A of the tuned model and this new sector B are then distributed according to a randomly generated pattern given in Table 4. The first nonlinear complex mode of each sector type is computed and used to build two different nonlinear superelements, which are then assembled in accordance with the mistuning pattern to build the reduced-order model.

The response of the mistuned system is computed for a travelling wave excitation with 6 nodal diameters, at a strong nonlinear level, and compared to the reference on Figure 5. As a consequence of the mistuning pattern, the response non longer exhibits one single peak, the 


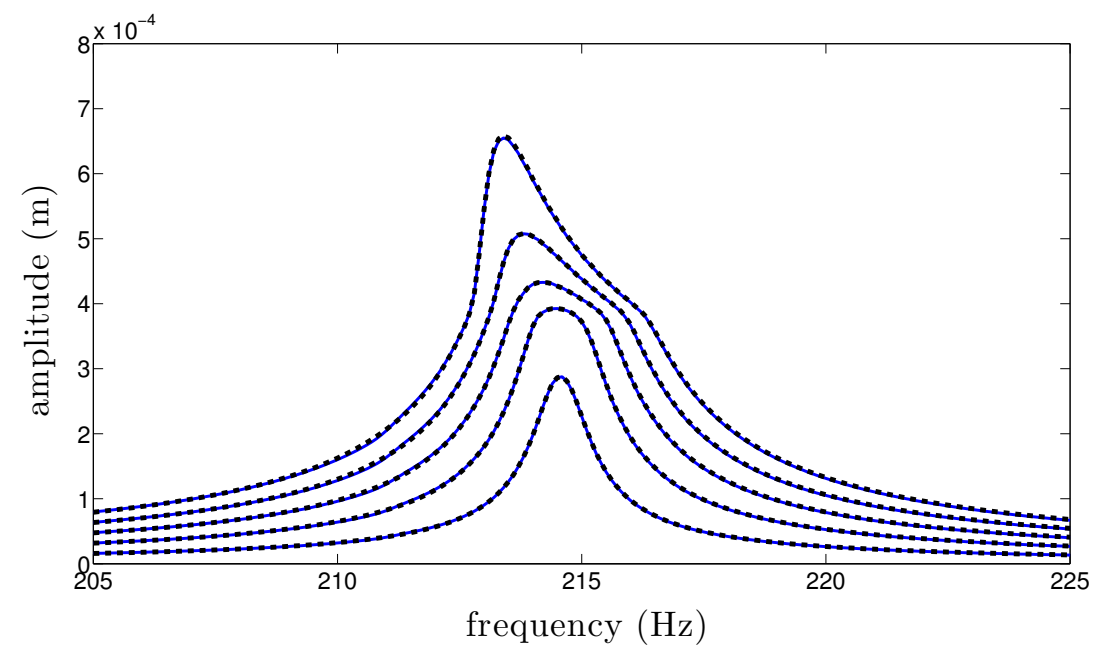

Figure 4: Response to a travelling wave excitation with 6 nodal diameters, for 5 excitation amplitudes (CNCMS in blue solid lines and HBM in black dashed lines)

\begin{tabular}{|l|ccccc|}
\hline$-($ unit $)$ & Tip & Middle & Root & Disk & Ground \\
\hline $\mathrm{m}(\mathrm{kg})$ & 0.2 & 0.3 & 0.4 & 1.2 & - \\
$\mathrm{k}\left(10^{6} \cdot \mathrm{N} / \mathrm{m}\right)$ & 1.8 & 0.9 & 40 & 50 & 0.6 \\
\hline
\end{tabular}

Table 3: Values of the parameters of sector B

\begin{tabular}{|l|cccccccccccc|}
\hline Sector & 1 & 2 & 3 & 4 & 5 & 6 & 7 & 8 & 9 & 10 & 11 & 12 \\
\hline Type & A & B & A & A & B & B & B & A & A & B & A & B \\
\hline
\end{tabular}

Table 4: Mistuning pattern 
travelling wave exciting now all the modes in the frequency range. Even though some small differences can be observed, the accuracy of the solution synthesised by CNCMS is very satisfactory. The origin of these differences is adressed in Section 4.2, and should be put in perspective with the complexity of the response and the computational efficiency of the method, discussed in Section 4.1 .

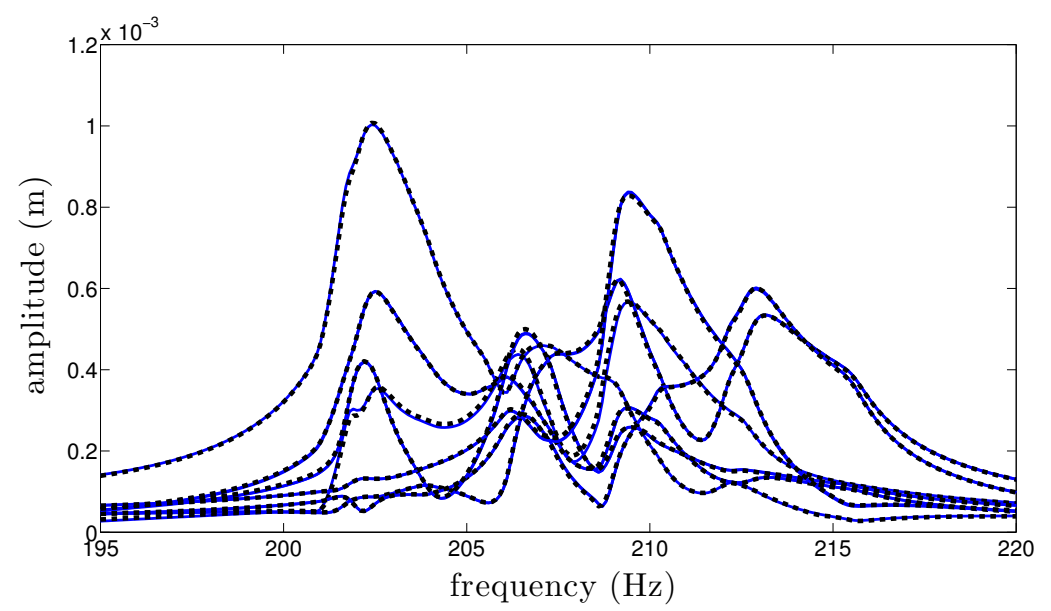

Figure 5: Response of all the blades of the mistuned system for a travelling wave excitation with 6 nodal diameters (CNCMS in blue solid lines, HBM black dashed lines)

\section{DISCUSSION}

This section briefly discusses the performance of the proposed reduced-order modeling technique. The efficiency of the method in terms of computational effort is first appraised, as well as the actual order-reduction obtained. The range of validity of the assumptions made is also adressed.

\subsection{Performance}

Figure 6 shows the ratio of the time required to compute the solution on a model with $72 \mathrm{sec}$ tors by HBM, normalized by the time required by CNCMS, for different excitation amplitudes $F$. Even close to the linear domain $(F=1 \mathrm{~N})$, the CNCMS is about already 80 times faster than the HBM, and is up to 190 times faster when the system is extremely nonlinear $(F=10 \mathrm{~N})$. Close to the optimum damping point $(F=5 \mathrm{~N})$, mentioned in Section 2.1 and where it can be interesting to operate industrial systems, the ratio is close to 135 , which highlights the outstanding potential of the CNCMS. The local maximum observed for $F=4 \mathrm{~N}$ also points out that the pattern of the nonlinear effects, besides the excitation level, can impact the performance of standard HBM methods when compared to the CNCMS.

The computational efficiency of the method clearly stands out in this study. It should however be pointed out that on the relatively small lumped-parameter model used here, the reduction capability of the CNCMS was not fully taken advantage of. On a larger model, the reduction arising from the use of nonlinear complex modes, allowing to drop all internal DOFs, would indeed be significantly more effective, and the computational performance of the method might prove even more impressive. 


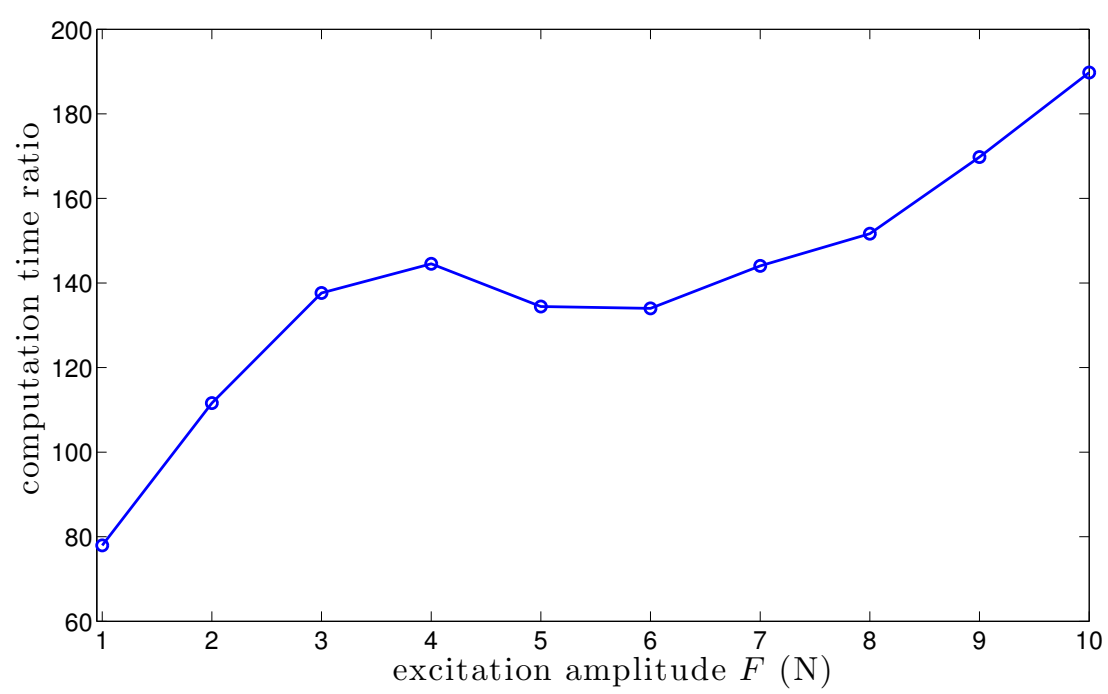

Figure 6: Computation time ratio between HBM and CNCMS

\subsection{Limitations}

In Section 3, some very small differences between the results obtained by CNCMS and the HBM reference could be observed. This could be explained by the fact that the model used here does not fully satisfy the assumptions made to derive the nonlinear superelement equations. In Eq. (6), the approximation of the displacement field for a given substructure uses indeed linear static modeshapes to account for the motion of the boundaries, which is quite understandable when it comes to actual blade-disk friction. On the lumped-parameter model of Section 3, however, the dry-friction elements are directly linked to the boundaries, which makes this approximation a bit bold. Nevertheless, the HBM reference curve is accurately approximated eventually, which allows to appraise the robustness of this new method.

\section{CONCLUSION}

The dynamic substructuring technique presented in this paper, the CNCMS, proved very promising for the study of forced vibrations in nonlinear structures. The method proved to handle quite well cases of neighboring resonant modes, and excellent computational performance has been obtained on a phenomenological model of mistuned bladed-disk, with a computation time about 135 times smaller than with standard and state-of-the-art HBM methods. Although devised initially in order to provide a new and efficient way to tackle problematics in bladed-disk dynamics, and allow to combine the presence of nonlinearities and mistuning, the procedure is flexible and could be applied to other types of nonlinear systems. Furthermore, the similarities between the CNCMS and the classic fixed-interface CMS perfectly allow to combine the two methods to build nonlinear and linear superelements, respectively, and build a hybrid reducedorder model by assembling these superelements.

Future prospects of this work could involve testing the procedure on other kind of nonlinearities, such as large deformation, or extend it to other CMS methods such as free-interface CMS. The implementation of the method on a large-scale 3D-model is an ongoing project of the authors, in view of assessing its potential for real, industrial structures. 


\section{REFERENCES}

[1] M. C. C. Bampton, R. R. Craig, Jr., Coupling of substructures for dynamic analyses, AIAA Journal 6 (7) (1968) 1313-1319. doi:10.2514/3.4741.

[2] R. Craig, C. J. Chang, Free-interface methods of substructure coupling for dynamic analysis, AIAA Journal 14 (11) (1976) 1633-1635. doi:10.2514/3.7264.

[3] D. D. Klerk, D. J. Rixen, S. N. Voormeeren, General framework for dynamic substructuring: History, review and classification of techniques, AIAA Journal 46 (5) (2008) 11691181. doi:10.2514/1.33274.

[4] K. Bathe, Finite Element Procedures, Prentice Hall, 2006.

[5] D. Laxalde, F. Thouverez, Complex non-linear modal analysis for mechanical systems: Application to turbomachinery bladings with friction interfaces, Journal of Sound and Vibration 322 (4-5) (2009) 1009-1025. doi:10.1016/j.jsv.2008.11.044.

[6] M. Krack, L. P. von Scheidt, J. Wallaschek, A method for nonlinear modal analysis and synthesis: Application to harmonically forced and self-excited mechanical systems, Journal of Sound and Vibration 332 (25) (2013) 6798-6814. doi:10.1016/j.jsv. 2013.08 .009 .

[7] D. S. Whitehead, Effect of mistuning on the vibration of turbo-machine blades induced by wakes, Journal of Mechanical Engineering Science 8 (1) (1966) 15-21. doi:10.1243/ JMES_JOUR_1966_008_004_02.

[8] J. T. Wagner, Coupling of turbomachine blade vibrations through the rotor, Journal of Engineering for Gas Turbines and Power 89 (4) (1967) 502. doi:10.1115/1.3616718.

[9] R. C. F. Dye, T. A. Henry, Vibration amplitudes of compressor blades resulting from scatter in blade natural frequencies, Journal of Engineering for Power 91 (3) (1969) 182187. doi:10.1115/1.3574726.

[10] D. Ewins, The effects of detuning upon the forced vibrations of bladed disks, Journal of Sound and Vibration 9 (1) (1969) 65-79. doi:10.1016/0022-460X (69) 90264-8.

[11] M. P. Castanier, C. Pierre, Modeling and analysis of mistuned bladed disk vibration: Current status and emerging directions, Journal of Propulsion and Power 22 (2) (2006) 384396. doi:10.2514/1.16345.

[12] M. P. Castanier, G. Óttarsson, C. Pierre, A reduced order modeling technique for mistuned bladed disks, Journal of Vibration and Acoustics 119 (3) (1997) 439-447. doi:10. $1115 / 1.2889743$.

[13] R. Bladh, M. P. Castanier, C. Pierre, Reduced order modeling and vibration analysis of mistuned bladed disk assemblies with shrouds, Journal of Engineering for Gas Turbines and Power 121 (3) (1999) 515-522. doi:10.1115/1.2818503.

[14] M. T. Yang, J. H. Griffin, A reduced-order model of mistuning using a subset of nominal system modes, Journal of Engineering for Gas Turbines and Power 123 (4) (1999) 893900. doi:10.1115/1.1385197. 
[15] M. Mbaye, C. Soize, J.-P. Ousty, E. Capiez-Lernout, Robust analysis of design in vibration of turbomachines, Journal of Turbomachinery 135 (2) (2012) 021008-021008. doi: $10.1115 / 1.4007442$.

[16] M. Krack, L. P. von Scheidt, J. Wallaschek, On the computation of the slow dynamics of nonlinear modes of mechanical systems, Mechanical Systems and Signal Processing 42 (1-2) (2014) 71-87. doi:10.1016/j.ymssp.2013.08.031.

[17] G. Kerschen, M. Peeters, J. Golinval, A. Vakakis, Nonlinear normal modes, part i: A useful framework for the structural dynamicist, Mechanical Systems and Signal Processing 23 (1) (2009) 170-194. doi:10.1016/j.ymssp.2008.04.002.

[18] C. Joannin, B. Chouvion, F. Thouverez, M. Mbaye, J.-P. Ousty, Nonlinear modal analysis of mistuned periodic structures subjected to dry friction, Journal of Engineering for Gas Turbines and Powerdoi:10.1115/1.4031886.

[19] T. M. Cameron, J. H. Griffin, An alternating frequency/time domain method for calculating the steady-state response of nonlinear dynamic systems, Journal of Applied Mechanics 56 (1) (1989) 149-154. doi:10.1115/1.3176036. 